\title{
Parameter Estimation of Polyphase Code Signals Based on Periodic FRFT
}

\author{
Jiandong Zhu*, Tengfei Jia, Yanfei Wei, Zhiqiang Ji, Youyi Fan and Junkui Xu \\ Electronic Test Equipment Center, Luoyang 471003, China. \\ zhujiandong82@163.com
}

\begin{abstract}
In order to improve the ability to intercepting polyphase code continuous wave signals for the radar reconnaissance receiver, a polyphase code continuous wave signals detection algorithm based on Periodic Fractional Fourier transform (Periodic FRFT, PFRFT) is proposed. In this paper, the PFRFT and its properties of linear frequency modulated continuous wave signals are studied. And the characteristics of linear frequency modulated continuous wave (LFMCW) signals of polyphase code continuous wave signals is analyzed. According to the similarity between the polyphase code continuous wave signals and the linear frequency modulated continuous wave signals, a polyphase code continuous wave signals detection algorithm based on PFRFT is proposed, also the realization process is given, and then the detection performance and parameter estimation performance are analyzed. Simulation results show that, compared with other existing algorithms, the proposed algorithm has better ability to detecting weak signal, and the minimum detectable signal to noise ratio (SNR) can be reduced by at least $6 \mathrm{~dB}$, which is further reduced with the observation time extended.
\end{abstract}

Keywords: Radar reconnaissance; polyphase code; continuous wave; signal detection; Periodic FRFT.

\section{Introduction}

The polyphase code continuous wave signals have the characteristics of phase jump and the time frequency characteristic like the LFMCW signal [1]. The polyphase code signals (i.e. Frank code, P1 code, P2 code, P3 code, P4 code) exported by LFMCW signals, take advantages of LFMCW signals and phase code signals. Compared with LFMCW signals, the polyphase code signals can obtain the lower self-correlation function side lobe level without the receiver weighting [2]. Compared with BPSK code signals, they have large Doppler tolerance. In addition, their modulation style is so complex to identify.

The polyphase code signals are quasi-LFMCW signals, the algorithm of these signal detection and estimation based on Radon-Wigner transform is proposed in the paper [3, 4]. The polyphase code signals parameter estimation using RAT is studied in [5], but it were not involved in carrier frequency estimation, because the RAT cannot be used to estimate carrier frequency of the LFM signals. When the modulation of the received signals and the reference signals is consistent, the result of conjugate multiplication between the received signals and the reference signals is a sine signal, which is used to detect and estimate the polyphase code signals [6].

The above methods do a lot of meaningful work in the detection and parameter estimation of the polyphase code continuous wave signals. In fact, the quasi-LFMCW signals are equivalent to a combination of a number of quasi-LFM pulse signals in these papers above. The algorithms of great performances in detecting the LFM pulse signals are used to detect quasi-LFM signals. Then a number of quasi-LFM signals detection algorithms are used to detect quasi-LFMCW signals. There are three problems in those methods mentioned above: The first one is the detection ability, which only stays on the ability of processing one single-pulse LFM and has poor performance on weak signal detection. The second one is LFM pulse signals bring the cross terms in the transform domain, which 
Seriously affect signal detection and estimation. The last one is that, there are manymixed pulses, which bring great difficulties to the identification of the overlapping signal.

In 2010, a periodic WHT algorithm [7] combining WHT and coherent processing was proposed by Francis G.Geroleo, which was applied to detect LFMCW signals in condition of low signal to noise ratio [8]. Based on the same thought, periodic FRFT algorithm [9, 10] improved from FRFT by redesigning its matching function to realize the quasi-coherent accumulation of LFMCW signal. In addition, it improved the detection ability of weak LFMCW signal, and avoided the cross item in the above method. So it is more suitable for the detection and estimation of LFMCW signal. In this paper, the periodic FRFT is applied to the polyphase code continuous wave signals, which will have greater effect than FRFT on the detection and estimation.

\section{Polyphase code and its periodic FRFT}

In order to match the LFMCW signals in the whole observation time, the FRFT kernel function need to be redesigned. Considering the time-frequency characteristics of LFMCW signal, the designed kernel function of periodic FRFT is [10],

$$
K_{\tilde{\alpha}}(t, \tilde{\tau}, \tilde{u}, \tilde{T})= \begin{cases}\sqrt{\frac{1-j \cot \tilde{\alpha}}{2 \pi}} \exp \left(j \frac{\bmod (t+\tilde{\tau}, \tilde{T})^{2}+\tilde{u}^{2}}{2} \cot \tilde{\alpha}\right. \\ -j(t+\tilde{\tau}) \tilde{u} \csc \tilde{\alpha}) & \tilde{\alpha} \neq n \pi \\ \sum_{n=-\infty}^{\infty} \delta(t+\tilde{\tau}-\tilde{u}-n \tilde{T}) & \tilde{\alpha}=2 n \pi \\ \sum_{n=-\infty}^{\infty} \delta(t+\tilde{\tau}+\tilde{u}-n \tilde{T}) & \tilde{\alpha}=(2 n \pm 1) \pi\end{cases}
$$

Where $\tilde{\tau}$ represents time offset search parameter and $\tilde{T}$ represents modulation period search parameter. The parameters set of the periodic FRFT kernel function is $\tilde{\Omega}=(\tilde{\alpha}, \tilde{u}, \tilde{T}, \tilde{\tau})$.Compared with the kernel function of FRFT, two dimensions parameters add to the kernel function of periodic FRFT, which are used to search for unknown parameters ( $\tau$ and $T$ ) of LFMCW signal. The periodic FRFT of $x(t)$ is defined as,

$$
\operatorname{PFRFT}[x(t)]=\int_{-\infty}^{+\infty} x(t) K_{\tilde{\alpha}}(t, \tilde{\tau}, \tilde{u}, \tilde{T}) d t
$$

The complex form of the phase code signals of the LPI radar can be expressed as,

$s(t)=A \exp \left[j\left(2 \pi f_{0} t+\varphi_{k}\right)\right]$

In the formula, $A$ is signal amplitude. $f_{0}$ is signal carrier frequency. $\varphi_{k}$ is phase code sequence with time variation, which is depended on the type of phase code modulation.

The polyphase code signal are derived from the LFM signals. In one code period $T$, the number of symbols is $N_{c}$, and chip width is $t_{b}$. Then the code period and bandwidth of the signal are respectively,

$$
\begin{aligned}
& T=N_{c} t_{b} \\
& B=1 / t_{b}
\end{aligned}
$$

The complex envelope of the transmitted signal in one code cycle can be expressed as,

$$
S(t)=A \sum_{m=1}^{N_{c}} c_{k} r e c t\left[\frac{t-(k-1) t_{b}}{t_{b}}\right]
$$

Where $c_{k}=\exp (j \varphi)$ and $\operatorname{rect}(\square)$ represents rectangular window.

Suppose that the number of symbols $N_{c}=64$, the carrier frequency $f_{0}=1 M H z$, sampling frequency $f_{s}=7 \mathrm{MHz}$, the signal amplitude $\mathrm{A}=1$, and the observation period contains 4 coding cycles. Figure 1 shows the smoothed pseudo Wigner distribution of LFMCW, Frank code, P1 code, P2 code, P3 code and P4 code. 


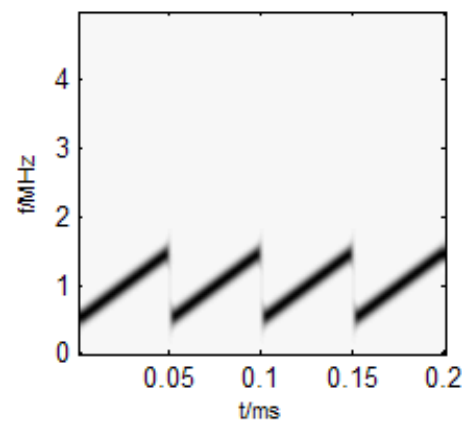

(a) LFMCW

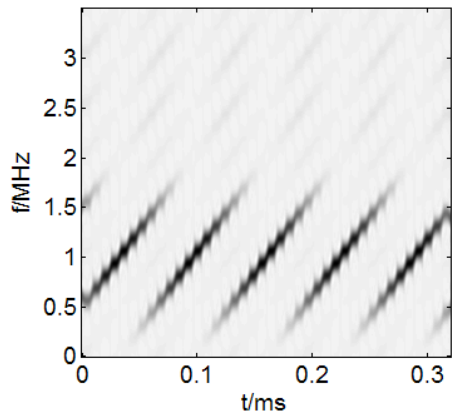

(c) P1

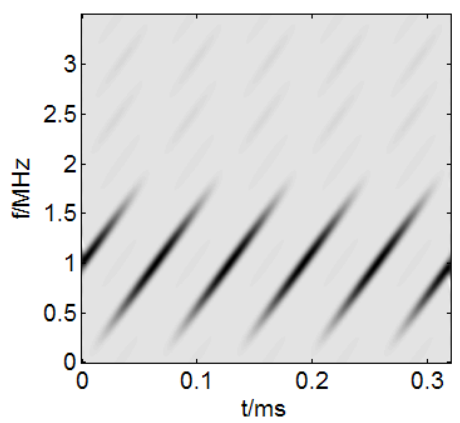

(e) P3

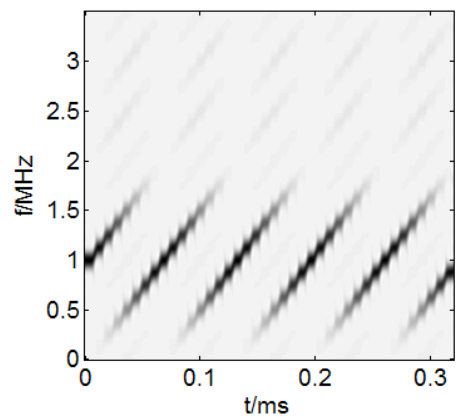

(b) Frank

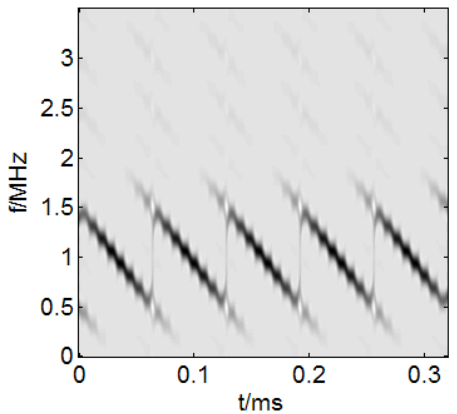

(d) P2

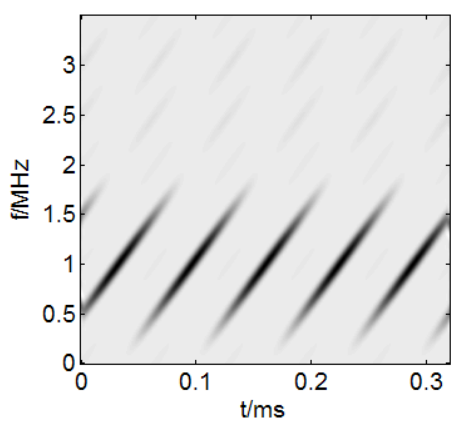

(f) $\mathrm{P} 4$

Fig. 1 SPWVD time frequency distribution of polyphase code signals

It can be seen from Fig. 1 that the time frequency image of the polyphase code signal is similar to the LFMCW, which shows the obvious periodic LFM feature. At the same time, there are both similarities and differences between the polyphase code signal and the LFMCW signal, mainly reflected on the following aspects.

(1) The time-frequency distributions of LFMCW signal and P4 code signal are the most similar. They both have obvious main ridge line, and their starting frequencies are located at zero point. The difference between them is that time-frequency distribution of $\mathrm{P} 4$ code signal has more cross terms, and due to the influence of coding, spectrum have leaked outside the coding start point.

(2) The time-frequency distributions of Frank code and P3 code signal both have obvious main ridge line, which has the same time-delay. Their time delay is about $1 / 2$ coding period, and at the same time, spectrum have leaked outside the coding start point.

(3) Compared to other polyphase code signals, the most obvious characteristic of P2 code signal is that the frequency modulation slope is negative. Its main ridge line is similar to the LFMCW signal with the negative frequency modulation slope. Due to the influence of coding, its spectrum have leaked outside the coding start point, which is slightly better than Frank code, P3 code and P4 code.

After application of the periodic FRFT to the above-mentioned polyphase code signals, the slices of periodic FRFT can be selected in $(\tilde{T}, \tilde{\tau})=(T, \tau)$, which describes the periodic FRFT features of the polyphase code signals. Fig.2 lists the slices of the periodic FRFT for LFMCW signals and various polyphase code signals, which are selected in $(\tilde{T}, \tilde{\tau})=(T, \tau)$. 


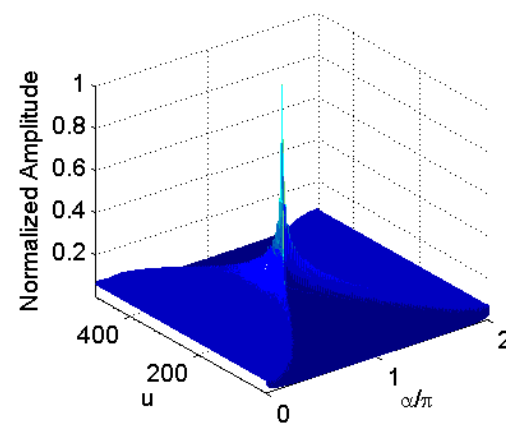

(a) LFMCW

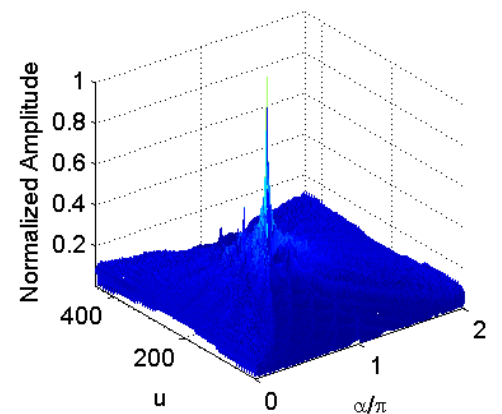

(c) P1

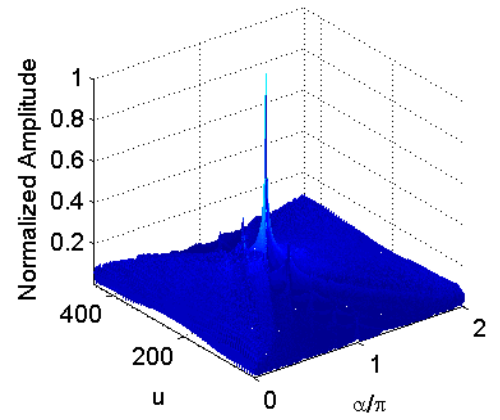

(e) P3

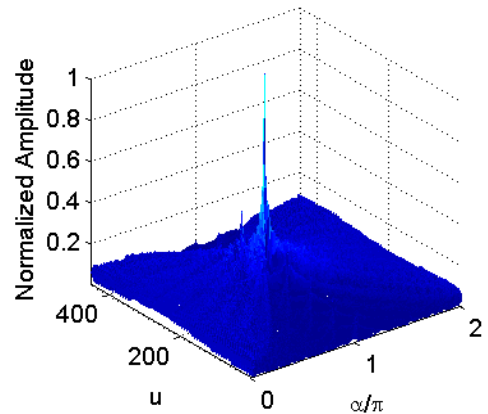

(b) Frank

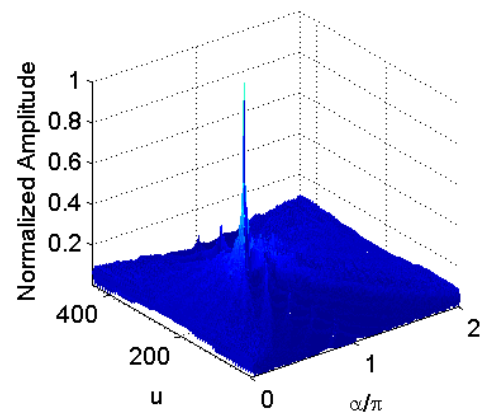

(d) P2

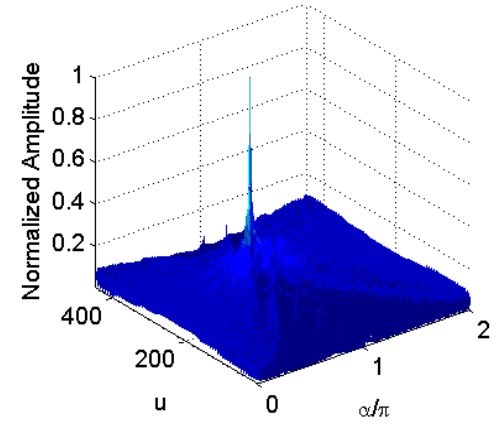

(f) $\mathrm{P} 4$

Fig. 2 The periodic FRFT of Polyphase code signals

Fig.2 shows that the polyphase code signals are similar to LFMCW signals and their periodic FRFT slices generate significant peaks. Affected by coding, the energy is not entirely concentrated on the main ridge line, and there is a loss of energy on accumulation, which is expressed as a smaller peak at other locations on the $(\alpha, u)$ plane. Therefore, the periodic FRFT has the effect of quasi-coherent accumulation on the polyphase code signals, which is used to detect and estimate polyphase code signals in the condition of low signal-to-noise ratio.

Take advantage of this property, the polyphase code radar signals of quasi-LFMCW signals are detected by detecting the peaks in the periodic FRFT domain. The peak point in the periodic FRFT domain is also the results of quasi-coherent accumulation of the main ridge line of LFM in the periodic FRFT domain. In theory, the method has stronger ability of detecting weak signal compared with the existing detection algorithms, and with the increase of the observation period, the number of the received polyphase code pulse is increased, so the detection ability is also enhanced.

\section{Detection and estimation of polyphase code signals}

\subsection{Detection and parameter estimation algorithm}

\section{Suppose}

$$
\left\{\begin{array}{l}
H_{0}: x(n)=w(n) \\
H_{1}: x(n)=s(n)+w(n)
\end{array}\right.
$$


Where $w(n)$ represents noise, and $s(n)$ represents polyphase code signal independent of noise. The problem of detecting polyphase code continuous wave signals in periodic FRFT domain, would be solved as follows. Firstly, the periodic FRFT of the input signal $x(n)$ is calculated. And then $H_{0}$ or $H_{1}$ is selected by comparing the periodic FRFT with the preset threshold $\beta$. If the value of the detected peak $\left(\left|\operatorname{PFRFT}_{\max }(\tilde{\alpha}, \tilde{u}, \tilde{T}, \tilde{\tau})\right|\right)$ exceeds the preset threshold $\beta$ in periodic FRFT domain, $H_{1}$ is true. Conversely, there is no polyphase code signal and $H_{0}$ is true. The detection algorithm is the same as the previous LFMCW detection algorithm.

The estimated parameters obtained by the periodic FRFT detection algorithm are limited to the parameter set $(\tilde{\alpha}, \tilde{u}, \tilde{T}, \tilde{\tau})$. This special set can be fully characterized by a LFMCW signal or a STLFMCW signal, but it can't describe the parameters of polyphase code signals. According to the feature parameter sets $(\tilde{\alpha}, \tilde{u}, \tilde{T}, \tilde{\tau})$, the other important parameters of the polyphase code signals can be obtained, including the signal bandwidth of $\mathrm{B}$, chip width of $t_{b}$, symbol number of $N_{c}$ and carrier frequency of $f_{0}$.

Chip width of the polyphase code signals is the reciprocal of the signal bandwidth, so

$t_{b}=\frac{1}{B}=\frac{1}{g T}$

The symbol number $N_{c}$ is the result of a modulation period divided by the chip width, namely,

$N_{c}=g T^{2}$

The carrier frequency $f_{0}$ can be obtained by the following formula.

$f_{0}=f_{i}+\frac{g T}{2}$

\subsection{The detection and parameter estimation flow}

The detection and parameter estimation of polyphase code continuous wave signals based on periodic FRFT, which is in the condition of additive Gauss white noise, are described in detail.

Step1: The signals are processed by decimating the mean value.

$x_{1}(n)=x(n)-\operatorname{mean}[x(n)]$

Step2: Set parameter search interval of $|\operatorname{PFRFT}(\tilde{a}, \tilde{u}, \tilde{T}, \tilde{\tau})|$ and calculate $|\operatorname{PFRFT}(\tilde{a}, \tilde{u}, \tilde{T}, \tilde{\tau})|$ of the signal received by the radar reconnaissance receiver.

Step3: Search for the peak of discrete periodic FRFT in the domain of $(\tilde{a}, \tilde{u}, \tilde{T}, \tilde{\tau})$.

Step4: According to the detected peak coordinates, use the preceding formula (8)-(10) for parameter estimation.

\subsection{Performance analysis of polyphase code signals detection}

The output signal to noise ratio (SNR) is the signal detection performance evaluation index. As a periodic FRFT result of the polyphase code signal is complex, its analytical form is difficult to get like [10], which would be difficult to analyze the detection performance of polyphase code signals in theory. A large number of simulations were proved that [5], the Radon-Ambiguity Transform(RAT) peak and the Radon-Wigner Transform (RWT) peak of polyphase code signals can obtain a fixed proportion of relationships, compared to the LFM signals with same parameters. Through abundant simulation experiments, both the peak value of FRFT and periodic FRFT for polyphase code signals detection have a fixed proportion. However, different from the above two kinds of transformation ratio, they are $0.55,0.58,0.70,0.60$ and 0.61 , respectively. It is illustrated that the detection performance of FRFT and FRFT is different than that of RAT and RWT.

From the previous analysis, in terms of LFMCW signal with the amplitude of 1, when in the condition of complex Gauss white noise, the output SNR of periodic FRFT detector to LFMCW signals is [10], 


$$
S N R_{i n}=\frac{N^{2} S N R_{i n}^{2}}{2 S N R_{i n}+1}
$$

The output SNR of the periodic FRFT detector to the polyphase code signals is,

$$
S N R_{i n}=\frac{\xi^{2} N^{2} S N R_{i n}^{2}}{2 S N R_{i n}+1}
$$

where $\xi=0.55,0.58,0.70,0.60,0.61$. Suppose that the input SNR of LFMCW signals and polyphase code signals is $S N R_{\text {in } 1}$ and $S N R_{\text {in } 2}$ respectively, and the output SNR of them is $S N R_{\text {out } 1}$ and $S N R_{\text {out } 2}$ respectively. By formula (9) and (10), $S N R_{\text {out } 1}=S N R_{\text {out } 2}$ should be met to achieve the same detection probability of the above two kinds of signals. When $N S N R_{i n} \square 1$, the input SNR (expressed in logarithmic terms) of them is,

$$
\left(S N R_{i n 1}-S N R_{i n 2}\right) d B=20 \lg \xi
$$

Because of $\xi<1$, the input SNR of the polyphase code continuous wave signals is higher than that of the LFMCW signals to achieve the same detection capability. Based on the large number of simulation experiments, the proportion of signal peak value is concluded, which is between the polyphase code signals of FRFT and periodic FRFT and the LFMCW signals. For the polyphase code signals with the same parameters, the ability of detecting P2 code using FRFT and periodic FRFT is the best, and the ability of detecting the other four kinds of polyphase code signals is relatively close. Among them, the ability of detecting Frank code is the worst. Also the ability of detecting P2 code is more $2 \mathrm{~dB}$ than the ability of detecting Frank code.

\section{Simulation experiment and analysis}

The detection and parameter estimation performance of the multi phase coded signals based on periodic FRFT are simulated and verified. The simulation parameters are set as follows. Suppose the additive Gauss white noise received in the polyphase code signals is independent of the signal. Set the symbol number $N_{c}=64$, the carrier frequency $f_{0}=1 \mathrm{MHz}$, the sampling frequency $f_{s}=7 \mathrm{MHz}$. The observation time includes four code period.

\subsection{Performance analysis of detection}

The detection performance of the algorithm is described by the relationship curves between the detection probability and the input SNR with the given false alarm probability.

The detection probability of five kinds of polyphase code signals, with the variation of the SNR, is shown in Fig.3. In order to analyze the advantage of periodic FRFT detection performance, the FRFT detection curves of the polyphase code signals are also given.

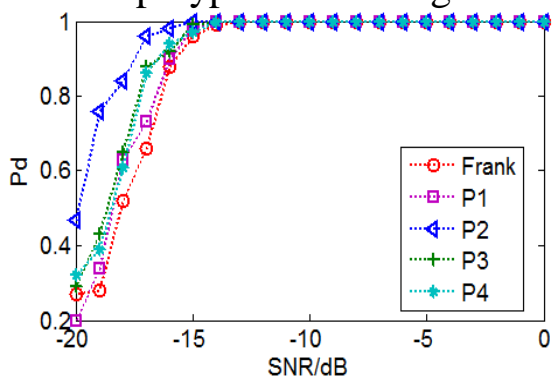

(a) Period FRFT

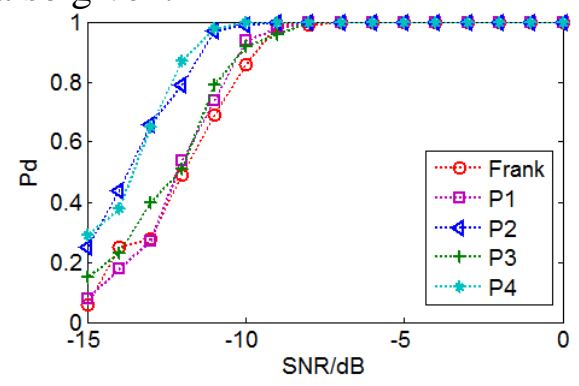

(b) FRFT

Fig. 3 The FRFT and FRFT detection probability for polyphase code signals

Fig. 3 shows that the periodic FRFT method proposed in the paper has a good detection ability for the polyphase code signals when the input SNR is about $-15 \mathrm{~dB}$, however, the traditional FRFT method has a good detection ability when the input SNR is about -9dB. Table 1 lists the required input SNR conditions of two detection methods when detection probability of polyphase code signals is $100 \%$.

The simulation results verify the weak signal detection ability of the polyphase code signals detection algorithm based on the periodic FRFT. 
Table 1 The required input SNR when the detection probability is $100 \%$

\begin{tabular}{|c|c|c|c|c|c|}
\hline & Frank & P1 & P2 & P3 & P4 \\
\hline PFRFT & $-14 \mathrm{~dB}$ & $-15 \mathrm{~dB}$ & $-16 \mathrm{~dB}$ & $-15 \mathrm{~dB}$ & $-15 \mathrm{~dB}$ \\
\hline FRFT & $-8 \mathrm{~dB}$ & $-9 \mathrm{~dB}$ & $-10 \mathrm{~dB}$ & $-9 \mathrm{~dB}$ & $-10 \mathrm{~dB}$ \\
\hline
\end{tabular}

\subsection{Performance analysis of parameter estimation}

The variation of the normalized mean square error (NRMSE) with the input SNR is analyzed, and also compared with the FRFT parameter estimation method. Signal parameters are set as before. The results of periodic FRFT and FRFT parameter estimation for five kinds of polyphase code signals are shown in Fig.4 and Fig.5.

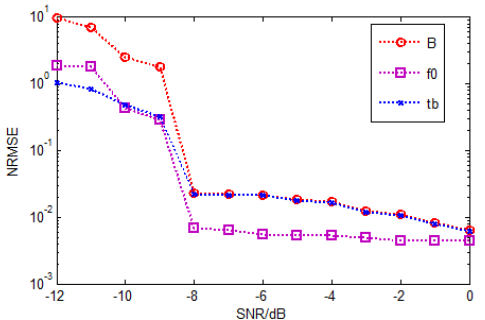

(a) Frank

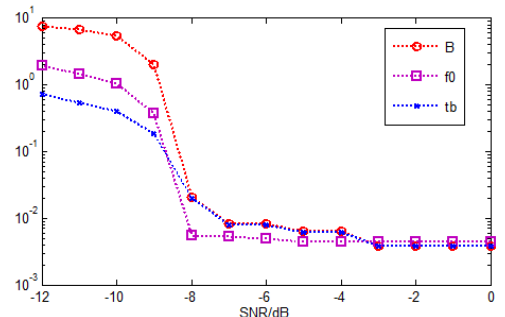

(b) P1

Fig.4 FRFT parameter estimation results of polyphase code signals

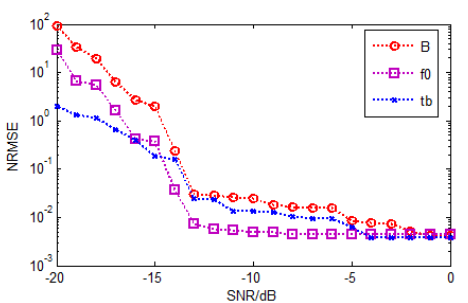

(a) Frank

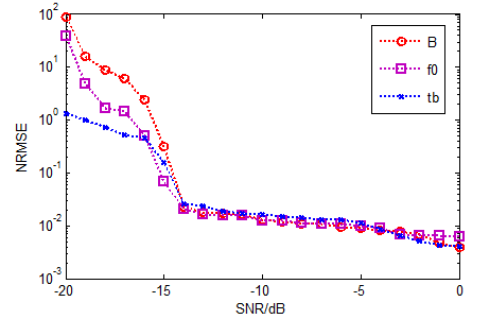

(b) P1

Fig. 5 Periodic FRFT parameter estimation results of polyphase code signals

It can be seen from Fig. 4 and Fig. 5, with the increase of the SNR, the estimation accuracy of the polyphase code signals is more and more high. FRFT has a very good parameter estimation effect on the polyphase code signals when the input SNR is more than $-9 \mathrm{~dB}$. However, periodic FRFT has a very good parameter estimation effect when the input SNR is greater than about $-15 \mathrm{~dB}$. The simulation results are same as that of the previous detection results. And the performance of the polyphase code signals, using the periodic FRFT to estimate, obtains about more $6 \mathrm{~dB}$ of the signal processing gain than that of FRFT.

\section{Conclusion}

In this paper, the quasi-coherent accumulation of LFMCW signals using periodic FRFT is extended to the application of polyphase code signals. The problems of detection and estimation for polyphase code signals are analyzed. Innovative points and main work are summarized as follows.

(1) The detection and parameter estimation of polyphase code continuous wave signals based on periodic FRFT are studied. It is concluded that the main ridge lines of Frank code, P1 code, P2 code, P3 code and P4 code continuous wave signal in the frequency domain are similar to characteristics of LFMCW. A detection and parameter estimation algorithm for them based on periodic FRFT is proposed, also the realization process is given, and then the detection performance and parameter estimation performance are analyzed.

(2) The detection and parameter estimation performance of the LFMCW continuous wave signals based on the periodic FRFT algorithm is verified by Monte-Carlo simulation. 


\section{Acknowledgments}

This work was financially supported by the National Natural Science Foundation of China (grant nos. 61401469 and 61301236).

\section{References}

[1] Benedetto J J, Konstantinidis I, Rangaswamy M. Phase-coded waveforms and their design [J]. IEEE Transaction Signal Processing Magazine, 2009: 23-30.

[2] Lee W K, Griffiths H D. Development of modified polyphase P codes with optimum sidelobe characteristics [J]. IEEE Proceedings Radar Sonar Navigation, 2004, 251(4):210-220.

[3] Pace P E. Detecting and classifying low probability of intercept radar [M].3rd ed. Norwood, MA: Horizon House Artech, 2009.

[4] Xu H Y, Zhou Y Y, Huang Z T. Detection and parameter estimation method for Frank code pulse compression waveforms [J]. Systems Engineering and Electronics, 2007, 29(6): 858-861.

[5] Xu H Y, Huang Z T, Zhou Y Y. Parameter Estimation Methods for Polyphase Codes Derived from LFM Waveforms using the Radon Transform [J]. Signal Processing, 2008, 24(2): 172-176.

[6] Deng Z M, Liu Y, Yang S S. Recognition of Polyphase-coded Radar Signals [J]. Journal of Data Acquisition and Processing, 2008, 23(3): 265-269.

[7] Geroleo F G and Pearce M B. Detection and Estimation of LFMCW Radar Signals [J]. IEEE Trans. on Aerospace and Electronic systems. 2012, 48(1): 405-417.

[8] Wang Z Z, Cao W P, Liu F. Code Continuous Wave Radar Signal Detection Algorithm Based on Periodic Wigner - Hough Transform [J]. Journal of Naval Aeronautical and Astronautical University, 2012, 27(6): 605-612.

[9] Huang Y, Liu F, Wang Z Z. Periodic FRFT-based LFMCW Signal Detection and Estimation [J]. Sci China Inf Sci, 2014, 44: 498-510.

[10]Zhu J D, Li J L, Gao X D. Adaptive Threshold Detection and Estimation of Linear Frequency Modulated Continuous - Wave Signals Based on Periodic Fractional Fourier Transform [J]. Circuit System and Signal Processing, 2016, 35(7): 2502-2517. 